\title{
STRONGLY ANALYTIC SPACES IN SPECTRAL DECOMPOSITION
}

\author{
by RIDGLEY LANGE and SHENGWANG WANG
}

(Received 30 May, 1986)

1. Introduction. It is now well-known that decomposable operators have a rich structure theory; in particular, an operator is decomposable iff its adjoint is [3]. There are many other criteria for decomposability [8], [9]. In Theorem 2.2 of this paper (see below) we give several new ones. Some of these (e.g. (ii), (iii)) are "relaxations" of conditions given in [7] and [8]. Assertion (vi) is a version of a result in [10]. Characterizations (iv) and (v) are novel in two respects. For instance, (v) states that an operator $T$ can be "patched" together into a decomposable operator if it has an invariant subspace $Y$ such that $T \mid Y$ and the coinduced operator $T / Y$ are both decomposable. Secondly, in this way the strongly analytic subspace appears in the theory of spectral decomposition.

Strongly analytic subspaces were introduced in [6], and Snader [13] characterized them as those $Y$ for which $T / Y$ has (Bishop) property $(\beta)$. One purpose of the present paper is to indicate an intimate connection between decomposable operators and strongly analytic subspaces. For example, one result that follows easily from [9, Th. 2.3 (iv)] is that if $T$ is decomposable then $T$ is strongly analytic iff $T / Y$ is decomposable. One result of the present paper (Corollary 3.10) is that two decomposable restrictions of the same operator can be "patched" together into a decomposable operator on the full underlying Banach space if the intersection of the two subspaces is strongly analytic.

We also study strongly analytic subspaces relative to a subclass of decomposable operators that Shulberg calls "superdecomposable" [12]. We show by example that this subclass strictly contains strongly decomposable operators.

2. Equivalent conditions for decomposability. In this section we begin with some preliminaries basic to the paper. Let $X$ be a complex Banach space and let $T$ be a bounded linear operator on $X$. We say that $T$ has property $(k)$ if $T$ has the single-valued extension property (SVEP) and the linear manifold $X_{T}(F)$ is closed if $F$ is a closed set of the complex plane $\mathbb{C}$ (see [3] for details); $T$ has property $(\beta)$ [2] if for any sequence $\left\{f_{n}: G \rightarrow X\right\}$ of analytic functions such that $(\lambda-T) f_{n}(\lambda) \rightarrow 0$ uniformly on each compact set in $G$ it follows that $f_{n} \rightarrow 0$ on such compacta also. It is easy to see that $T$ has property $(k)$ whenever it has property $(\beta)$.

Now let $Y$ be a $T$-invariant subspace. As usual, $T \mid Y$ denotes the restriction to $Y$, while $T / Y$ denotes the operator coinduced by $T$ on the quotient space $X / Y$. In [6] $Y$ was defined to be strongly analytic for $T$ if for each sequence $\left\{f_{n}: G \rightarrow X\right\}$ of analytic functions such that

$$
\operatorname{dist}\left((\lambda-T) f_{n}(\lambda), Y\right) \rightarrow 0
$$

Glasgow Math. J. 30 (1988) 249-257. 
uniformly on compacta in $G$ it also happens that

$$
\operatorname{dist}\left(f_{n}(\lambda), Y\right) \rightarrow 0
$$

uniformly on compacta in $G$. Later Snader proved [13] that $Y$ is strongly analytic iff $T / Y$ has property $(\beta)$.

We say $T$ has the asymptotic spectral decomposition (abbrev. (ASD)) if for each finite open cover $\left\{G_{i}\right\}_{i=1}^{n}$ of the plane, there exists a system of $T$-invariant subspaces $\left\{M_{i}\right\}_{i=1}^{n}$ such that

$$
X=\bigvee_{i=1}^{n} M_{i} \text { and } \sigma\left(T \mid M_{i}\right) \subset G_{i} \quad(1 \leqslant i \leqslant n) .
$$

A simpler version when $n=2$ will often suffice. For each open cover $\{G, H\}$ of $\mathbb{C}$, there are $T$-invariant subspaces $M, N$ such that

$$
X=M \vee N, \quad \sigma(T \mid M) \subset G, \quad \sigma(T \mid N) \subset H .
$$

If in $\left(^{*}\right), X=M+N$, then $T$ is decomposable by [7] and [11]. Moreover, every decomposable operator $T$ has property $(\beta)$ and so $X_{T}(F)$ is closed if $F$ is closed in $\mathbb{C}$. We also recall that $T$ is strongly decomposable if each restriction $T \mid X_{T}(F)$ ( $F$ closed) is decomposable. Finally, it is an open question whether $\left({ }^{*}\right)$ implies $(2.1)$ for all $n$.

A decomposable operator $T$ is said to be superdecomposable [12] if for closed $F$ and open $G, F \subset G \subset \mathbb{C}$, there exists a $T$-invariant subspace $M$ such that

$$
X_{T}(F) \subset M \subset X_{T}(G)
$$

and $T \mid M$ is decomposable. Obviously every strongly decomposable operator is superdecomposable.

2.1. Proposition. Given $T$, if $T \mid Y$ and $T / Y$ both have property $(\beta)$, then $T$ has property $(\beta)$.

Proof. Let $f_{n}: \omega \rightarrow X$ be a sequence of functions analytic on $\omega$ satisfying

$$
(\lambda-T) f_{n}(\lambda) \rightarrow 0
$$

uniformly in norm on each compact subset of $\omega$. Let $K=\left\{\lambda:\left|\lambda-\lambda_{0}\right|<r\right\}$ with $\bar{K} \subset \omega$ and expand $f_{n}$ as

$$
f_{n}(\lambda) \sum_{k=0}^{\infty} a_{n k}\left(\lambda-\lambda_{0}\right)^{k}
$$

on a neighborhood of $\bar{K}$. By (2.2) and the hypothesis on $T / Y, \hat{f}_{n} \rightarrow \hat{0}$ uniformly in the quotient norm of $X / Y$ on $\bar{K}$. Moreover, (2.3) implies that

$$
\hat{f}_{n}(\lambda)=\sum_{k=0}^{\infty} \hat{a}_{n k}\left(\lambda-\lambda_{0}\right)^{k}
$$

Put $E_{n}=\max _{\left|\lambda-\lambda_{0}\right|=r}\left\|\hat{f}_{n}(\lambda)\right\| ;$ then $E_{n} \rightarrow 0$. By the Cauchy inequality one has

$$
\left\|\hat{a}_{n k}\right\| \leqslant \frac{E_{n}}{r^{k}}
$$


or equivalently, $\operatorname{dist}\left(a_{n k}, Y\right) \leqslant E_{n} / r^{k}$. Now choose $b_{n k} \in Y$ such that

$$
\left\|a_{n k}-b_{n k}\right\| \leqslant 2 E_{n} / r^{k} \text {. }
$$

It follows from the resulting inquality

$$
\left\|b_{n k}\right\| \leqslant\left\|a_{n k}\right\|+\frac{2 E_{n}}{r^{k}}
$$

that the series

$$
g_{n}(\lambda)=\sum_{k=0}^{\infty} b_{n k}\left(\lambda-\lambda_{0}\right)^{k}
$$

converges in the disc $K$ and hence is analytic there. On the other hand, (2.2) and (2.4) imply that

$$
(\lambda-T) g_{n}(\lambda)=(\lambda-T)\left[g_{n}(\lambda)-f_{n}(\lambda)\right]+(\lambda-T) f_{n}(\lambda) \rightarrow 0
$$

uniformly in norm on each compact subset of $K$. Since $T \mid Y$ has property $(\beta)$ and $g_{n}(\lambda) \in Y$ for $\lambda \in K$, (2.5) implies that $g_{n} \rightarrow 0$ uniformly on each compact set in $K$. Then (2.4) implies

$$
f_{n}(\lambda) \rightarrow 0
$$

uniformly in norm (of $X$ ) on each compact set in $K$. By the Heine-Borel theorem (2.6) remains valid on every compact set in $\omega$. Thus $T$ has property $(\beta)$, and the proposition is proved.

2.2. THEOREM. For an operator $T$ the following are equivalent:

(i) $T$ is decomposable;

(ii) for every pair of open sets $G$ and $H$ with $\bar{G} \subset H$, there exists a T-invariant subspace $Y$ such that

$$
\sigma(T \mid Y) \subset \mathbb{C}-G, \quad \sigma(T / Y) \subset \bar{H} ;
$$

(iii) $T$ has property $(\beta)$ and $T^{*}$ has the SVEP such that for every open disc $D$ the spectral manifold $X_{T^{*}}^{*}(\mathbb{C}-D)$ is norm closed;

(iv) there exists a $T$-invariant subspace $Y$ such that $T \mid Y$ and $T / Y$ are both decomposable;

(v) there exists a $T$-invariant subspace $Y$ such that $T \mid Y$ and $T^{*} \mid Y^{\perp}$ are decomposable;

(vi) Thas property ( $k$ ) and for each open $G$,

$$
\sigma\left(T^{*} \mid X_{T}(G)^{\perp}\right) \subset \mathbb{C}-G .
$$

Proof. The conclusion will be reached through the sequences of implications

$$
\text { (i) } \Rightarrow \text { (ii) } \Rightarrow \text { (iii) } \Rightarrow \text { (i), (i) } \Leftrightarrow \text { (iv) } \Leftrightarrow \text { (v) and (i) } \Leftrightarrow(\text { vi). }
$$

(i) $\Rightarrow$ (ii). Let $G$ and $H$ be open sets such that $\bar{G} \subset H$. Since $T$ is decomposable, one 
has

$$
X=X_{T}(\mathbb{C}-G)+X_{T}(\bar{H}) .
$$

Let $Y=X_{T}(\mathbb{C}-G)$. Then $\sigma(T \mid Y) \subset \mathbb{C}-G$. Since $T / Y$ is similar to

$$
\left[T \mid X_{T}(\bar{H})\right] /\left[Y \cap X_{T}(\bar{H})\right],
$$

We see that $\sigma(T \mid Y) \subset \bar{H}$, hence $T$ satisfies (2.7).

(ii) $\Rightarrow$ (iii). Let $G, H$ be open discs such that $\bar{G} \subset H$. Then (2.7) implies [9, Theorem 2.3 (iii, a)].

Put $G_{1}=\mathbb{C}-\bar{H}, H_{1}=\mathbb{C}-\bar{G}$, so that $G_{1}, H_{1}$ are open sets with $\bar{G}_{1} \subset H_{1}$. It follows from (2.7) that there is a $T$-invariant subspace $Z$ such that

$$
\begin{aligned}
& \sigma(T \mid Z) \subset \mathbb{C}-G_{1}=\bar{H}, \\
& \sigma(T / Z) \subset \bar{H}_{1} \subset \mathbb{C}-G .
\end{aligned}
$$

and hence [9, Th. 2.3 (iii, b)] is satisfied. By [9, Th. 2.3], $T$ has property $(\beta)$ and $T^{*}$ has the SVEP such that $X_{T^{*}}^{*}(F)$ is closed for $F$ closed. In particular, $X_{T^{*}}^{*}(\mathbb{C}-D)$ is closed for each open disc $D$; hence (iii) holds.

(iii) $\Rightarrow$ (i) Since $X_{T^{*}}^{*}(\mathbb{C}-D)$ is closed, we have ${\overline{X_{T}(D)}}^{\perp}=X_{T^{*}}^{*}(\mathbb{C}-D)$ by [9, Theorem 2.3]: hence

$$
\sigma\left(T^{*} / X_{T^{*}}^{*}(\mathbb{C}-D)\right)=\sigma\left(T \mid \overline{\left.X_{T}(D)\right)} \subset \bar{D} .\right.
$$

From this and the evident inclusion

$$
\sigma\left(T^{*} \mid X_{T}^{*}(\mathbb{C}-D)\right) \subset \mathbb{C}-D
$$

one obtains, by [3, Theorem 5.8], that $T^{*}$ has property ( $\beta$ ). Applying [9, Theorem 2.3] again, we infer that $T$ is decomposable.

(i) $\Leftrightarrow($ iv) $\Leftrightarrow$ (v) Since it is easily seen that (iv) and (v) are equivalent, it suffices to prove (i) $\Leftrightarrow$ (iv).

If (i) holds, then $Y=\{0\}$ satisfies (iv). Conversely, suppose for some $T$-invariant $Y, T \mid Y$ and $T / Y$ are decomposable. Since it follows from [4] that $T \mid Y$ and $T / Y$ both have property $(\beta)$, by Proposition $2.1, T$ has property $(\beta)$. From the duality relations

$$
T^{*} \mid Y^{\perp}=(T / Y)^{*}, \quad T^{*} / Y^{\perp}=(T \mid Y)^{*}
$$

and [3, Theorem 8.1], it follows that $T^{*} \mid Y^{\perp}$ and $T^{*} / Y^{\perp}$ are both decomposable; hence both have property $(\beta)$ [4]. Again Proposition 2.1 implies that $T^{*}$ has property $(\beta)$. By [9, Theorem 2.3 (iv)] $T$ is decomposable.

(i) $\Leftrightarrow$ (vi). Since (i) $\Rightarrow$ (vi) is evident, we suppose that (vi) holds. We prove that, for every $T$-invariant subspace $Z$ satisfying $X_{T}(G) \subset Z$, one has

$$
\sigma\left(T \mid \overline{\left.X_{T}(G)\right)} \subset \sigma(T \mid Z)\right. \text {. }
$$

Let $x \in X_{T}(G)$ and let $x(\cdot)$ be the local resolvent of $T$ at $x$. For $\lambda \in \rho_{T}(x)$ (the local resolvent set) $x(\lambda) \in X_{T}(G)$ and $x=(\lambda-T) x(\lambda)$. Now let $\lambda_{0} \in \rho(T \mid Z)$. For $\lambda \in \rho_{T}(x)$

$$
(\lambda-T) R\left(\lambda_{0} ; T \mid Z\right) x(\lambda)=R\left(\lambda_{0} ; T \mid Z\right)(\lambda-T) x(\lambda)=R\left(\lambda_{0} ; T \mid Z\right) x
$$


and since the first expression is analytic on $\rho_{T}(x)$, it follows that

$$
\sigma_{T}\left(R\left(\lambda_{0} ; T \mid Z\right) x\right) \subset \sigma_{T}(x) \subset G,
$$

or, equivalently,

$$
R\left(\lambda_{0} ; T \mid Z\right) x \in X_{T}(G) .
$$

By (2.10) $\overline{X_{T}(G)}$ is invariant under $R\left(\lambda_{0} ; T \mid Z\right)$, so (2.9) follows by [3, Proposition 4.2]. In particular

$$
\sigma\left(T \mid \overline{\left.X_{T}(G)\right)} \subset \sigma\left(T \mid X_{T}(\bar{G})\right) \subset \bar{G} .\right.
$$

By (2.7), (2.11) and [3, Theorem 5.17] $T$ is decomposable. Theorem 2.2 is thus proved.

By means of Theorem 2.2(iv) we give a very easy proof of the following well-known fact.

2.3. CoRollary. If operators $T_{i}(i=1,2)$ are decomposable on Banach spaces $X_{i}$, then $T_{1} \oplus T_{2}$ is decomposable on $X_{1} \oplus X_{2}$.

Proof. Let $S=T_{1} \oplus T_{2}$. Then $S / X_{1}$ is similar to $T_{2}$ and is therefore decomposable. Since $S \mid X_{1}=T_{1}$ the result follows from Theorem 2.2(iv).

2.4 Remark. If $T \mid Y$ and $T / Y$ both have property $(\beta), T$ need not be decomposable. For example, let $T$ be right shift on the Hilbert space $X=l^{2}(\mathbb{N})$, and let $Y=T X$. Then $T \mid Y$ has property $(\beta)$ and $T / Y^{\prime}=0$ so it also has property $(\beta)$. But $T$ is not decomposable. This example also answers the following question negatively. If $T \mid Y$ has property $(\beta)$ and $T / Y$ is decomposable, then is $T$ decomposable? However, the following question is open. If $T / Y$ is decomposable and $T \mid Y$ has (ASD) with property $(\beta)$ then is $T$ decomposable?

3. Applications. In this section we prove some results in which strongly analytic subspaces play important roles in spectral decompositions. Theorem 3.3 generalizes and sharpens [8, Theorem 8] from the reflexive to the case of arbitrary Banach spaces. We also give some theorems complementing one of Shulberg [12] concerning superdecomposable operators. Most of these results involve strongly analytic subspaces. For example if $T$ is superdecomposable with strongly analytic subspaces (Definition 3.6), then $T^{*}$ is also. One interesting consequence of this set of ideas is the following. Although as remarked in $\$ 2$ every strongly decomposable operator is superdecomposable, the converse is false. Hence superdecomposable operators form a strictly larger class than strongly decomposable operators. Our last theorem (Theorem 3.9) is another result on "patching" together a decomposable operator from "parts", in which strong analyticity is an important hypothesis. Then

3.1. Proposition. Let $X=M \vee N$ with $M$ strongly analytic for $T$ and $N T$-invariant.

$$
\sigma(T / M) \subset \sigma(T \mid N)
$$


Proof. For every fixed $x \in X$ there exist sequences $\left\{y_{n}\right\}$ in $M$ and $\left\{z_{n}\right\}$ in $N$ such that

$$
x=\lim _{n \rightarrow \infty}\left(y_{n}+z_{n}\right) \text {. }
$$

Write $f_{n}(\lambda)=R(\lambda ; T \mid N) z_{n}$ for $\lambda \in \rho(T \mid N)$. Then $z_{n}=(\lambda-T) f_{n}(\lambda)$ and

$$
x=\lim _{n \rightarrow \infty}\left(y_{n}+(\lambda-T) f_{n}(\lambda)\right) \text { for each } \lambda \varepsilon \rho(T \mid N) .
$$

Let $\hat{T}=T / M$ and let $\hat{x}$ denote a coset in $X / M$. Then (3.2) and (3.3) imply

$$
\hat{x}=\lim _{n \rightarrow \infty}(\lambda-\hat{T}) \hat{f}_{n}(\lambda)=\lim _{n \rightarrow \infty} \hat{z}_{n}(\lambda \in \rho(T \mid N)) .
$$

Since in fact $\hat{z}_{n}=(\lambda-\hat{T}) \hat{f}_{n}(\lambda)$, (3.4) holds uniformly on $\rho(T \mid N)$. By the fact that $M$ is strongly analytic (i.e. $T / M$ has property $(\beta)$ ) one obtains that $\hat{f}_{n}(\lambda)$ converges to a function $\hat{f}(\lambda)$ uniformly on every compact subset of $\rho(T \mid N)$. Now (3.4) implies

$$
\hat{x}=(\lambda-\hat{T}) \hat{f}(\lambda) \quad(\lambda \in \rho(T \mid N)) ;
$$

hence $\lambda-\hat{T}$ is surjective for $\lambda \in \rho(T \mid N)$. Since $\hat{T}$ has the SVEP, by [3, Corollary 2.5] $\lambda \in \rho(T / M)$, hence (3.1) holds.

3.2. Proposition. Let $X=M \vee N$ with $M$ strongly analytic for $T$ and $N T$-invariant, and let $T$ have the SVEP. Then for every subset $E$ with $\sigma(T \mid N) \cap E=\varnothing$ one has $X_{T}(E) \subset M$.

Proof. For $x \in X_{T}(E)$, it follows from Proposition 3.1 and the inclusions $\sigma_{T / M}(\hat{x}) \subset$ $\sigma_{T}(x) \subset E$ that

$$
\sigma_{T / M}(\hat{x}) \subset \sigma(T / M) \cap E \subset \sigma(T \mid N) \cap E=\varnothing ;
$$

hence $\hat{x}=0$, or $x \in M$. This proves the desired inclusion.

The next theorem generalizes [8, Th. 8] to the case of arbitrary Banach spaces.

3.3. THEOREM. If $T$ has the (ASD) with either $M$ or $N$ in $\left(^{*}\right)$ strongly analytic for $T$, then $T$ is decomposable and $T^{*}$ is superdecomposable.

Proof. Let $G, H$ be open such that $\bar{G} \subset H$. By $\left({ }^{*}\right)$ there are $T$-invariant subspaces $M$ and $N$ with

$$
X=M \vee N, \quad \sigma(T \mid M) \subset \mathbb{C}-G, \quad \text { and } \quad \sigma(T \mid N) \subset \bar{H},
$$

where we suppose that $M$ is strongly analytic. By Proposition $3.1 \sigma(T / M) \subset \sigma(T \mid N) \subset \bar{H}$. Hence $T$ is decomposable by Theorem 2.2(ii). By [3, Theorem 8.1], $T^{*}$ is also decomposable. Now, let $F$ be closed and $G$ be open with $F \subset G$. Put $K=\mathbb{C}-G$, $H=\mathbb{C}-F$. Then $K$ is closed, $H$ is open and $K \subset H$. Since $\{G, H\}$ covers $\mathbb{C}$, there are $T$-invariant subspaces $M, N$ such $X=M \vee N, \sigma(T \mid M) \subset H$ and $\sigma(T \mid N) \subset G$ with $M$ strongly analytic. It follows that $M \subset \overline{X_{T}(H)}$, so

$$
X_{T}^{*}(F)={\overline{X_{T}(H)}}^{\perp} \subset M^{\perp} .
$$


But Proposition 3.1 implies that $\sigma(T / M) \subset \sigma(T \mid N) \subset G$, or equivalently, $\sigma\left(T^{*} \mid M^{\perp}\right) \subset$ G. Hence

$$
M^{\perp} \subset X_{T^{*}}^{*}(G)
$$

Since $M$ is strongly analytic for $T$, i.e. $T / M$ has property $(\beta)$, and $T^{*} \mid M^{\perp}\left(=(T / M)^{*}\right)$ also has property $(\beta)$ by restriction, we have that $T^{*} / M^{\perp}$ is decomposable by [9, Theorem 2.3]. Now (3.5) and (3.6) complete the proof that $T^{*}$ is superdecomposable.

Shulberg [12] proved that if $T$ is strongly decomposable, then $T^{*}$ is superdecomposable. By the predual theorem [3, Theorem 9.6] we can prove the "dual" of Shulberg's result.

\subsection{THEOREM. If $T^{*}$ is strongly decomposable, then $T$ is superdecomposable.}

Proof. Let $F$ be closed and $G$ be open with $F \subset G$, and let $H$ be open with $F \subset H \subset \bar{H} \subset G$. Since $T^{*}$ is strongly decomposable, $T^{*} / X_{T^{*}}^{*}(K)$ is decomposable, where $K=\mathbb{C}-H$, and hence $T \mid \overline{X_{T}(H)}$ is decomposable. Obviously $X_{T}(F) \subset \overline{X_{T}(H)} \subset X_{T}(G)$; hence $\left({ }^{* *}\right)$ is satisfied and the proof is complete.

3.5. Remark. By [14, Theorem 3], Albrecht's example [1] of a decomposable operator $T$ which is not strongly decomposable has adjoint that is strongly decomposable. Hence the predual $T$ is superdecomposable by Theorem 3.4. Moreover, $\overline{X_{T}(G)}$ is strongly analytic for $T$ for each open $G$, so $T$ also satisfies the hypothesis of Theorem 3.3. We can then infer the following:

(i) superdecomposable operators need not be strongly decomposable;

(ii) the hypotheses of Theorem 3.3 are not strong enough to guarantee that the operator is strongly decomposable.

Now two questions arise.

(1) Does decomposability imply superdecomposability?

(2) Is superdecomposability preserved under duality?

We give a partial answer to Question 2. To do this we introduce the following definition.

3.6. Definition. If $T$ is superdecomposable such that the intermediate space $M$ in $\left({ }^{* *}\right)$ may be chosen strongly analytic, then we say that $T$ is (sa)-superdecomposable.

3.7. THeоRem. If $T$ is (sa)-superdecomposable, then so is $T^{*}$.

Proof. Since $T$ evidently satisfies the hypotheses of Theorem 3.3, $T^{*}$ is superdecomposable. Let $M$ be the $T$-invariant subspace of Definition 3.6 , so that $T \mid M$ is decomposable and $M$ is strongly analtyic. By duality $T^{*} / M^{\perp}$ is decomposable; hence $M^{\perp}$ is strongly analytic. Now (3.5) and (3.6) above show that $M^{\perp}$ satisfies Definition 3.6, and so $T^{*}$ is (sa)-superdecomposable. The converse of Theorem 3.7 is not clear because the intermediate subspace in Definition 3.6 need not be weak* closed. But we do have the following sharpening of Theorem 3.4 . 
3.8. THEOREM. If $T^{*}$ strongly decomposable, then $T$ is (sa)-superdecomposable.

Proof. Referring to the proof of Theorem 3.4, we see that $T / \overline{X_{T}(H)}$ is decomposable since $T^{*} \mid X_{T^{*}}^{*}(K)$ is. Hence $\overline{X_{T}(H)}$ is strongly analytic for $T$, and thus $T$ is (sa)-superdecomposable.

Our next theorem generalizes Corollary 2.3.

3.9. THEOREM. Let $Y_{1}, Y_{2}$ be invariant under $T$. If

(i) $Y_{1}+Y_{2}$ is closed,

(ii) $T \mid Y_{i}$ is decomposable $(i=1,2)$,

(iii) $Y_{1} \cap Y_{2}$ is strongly analytic for $T\left(\right.$ or $\left.T \mid\left(Y_{1}+Y_{2}\right)\right)$,

then $T \mid\left(Y_{1}+Y_{2}\right)$ is decomposable.

Proof. Let $S=T \mid\left(Y_{1}+Y_{2}\right), Y=Y_{1} \cap Y_{2}$. By (ii) $S \mid Y_{i}(i=1,2)$ is decomposable, so it also has property $(\beta)$, and $S \mid Y$ has this same property by restriction. Since $Y$ is strongly analytic for $S$, again $S / Y$ has property $(\beta)$. By Proposition $2.1, S$ has property $(\beta)$.

Next we prove that $S^{*}$ has property $(\beta)$. Decomposability of $T \mid Y_{i}(i=1,2)$ implies that of $T^{*} / Y_{i}^{\perp}$ as well; hence $Y_{i}^{\perp}$ is strongly analytic for $T^{*}$. By [5, Theorem IV.4.8] $Y_{1}^{\perp}+Y_{2}^{\perp}$ is closed in $X^{*}$; hence, by [6, Proposition 7], $Y_{1}^{\perp} \cap Y_{2}^{\perp}$ is strongly analytic for $T^{*}$, and thus $T^{*} /\left(Y_{1}^{\perp} \cap Y_{2}^{\perp}\right)$ has property $(\beta)$. But

$$
S^{*}=\left[T \mid\left(Y_{1}+Y_{2}\right)\right]^{*}=T^{*} /\left(Y_{1}+Y_{2}\right)^{\perp}=T^{*} /\left(Y_{1}^{\perp} \cap Y_{2}^{\perp}\right) .
$$

Since we have thus shown that $S$ and $S^{*}$ have property $(\beta), S$ is decomposable by [9, Theorem 2.3(iv)].

3.10 Corollary. Let $Y_{1}, Y_{2}$ be invariant under $T$ such that

(i) $X=Y_{1}+Y_{2}$,

(ii) $T \mid Y_{i}$ is decomposable $(i=1,2)$,

(iii) $Y_{1} \cap Y_{2}$ is strongly analytic for $T$.

Then $T$ is decomposable and $Y_{1}(i=1,2)$ is strongly analytic for $T$.

Proof. $T$ is decomposable by Theorem 3.9. Since $T / Y_{i}$ is similar to $\left(T \mid Y_{j}\right) / Y_{1} \cap Y_{2}$, where $i, j=1,2$ and $i \neq j$, and by hypothesis $Y_{1} \cap Y_{2}$ is strongly analytic for $T$, $\left(T \mid Y_{j}\right) / Y_{1} \cap Y_{2}$ has property $(\beta)$, so that $T / Y_{i}$ also has property $(\beta)$, and hence $Y_{i}$ is strongly analytic for $T$.

3.11 Remark. The explicit statement of [6, Proposition 7] referred to in the proof of Theorem 3.9 is as follows. If $Y_{i}(i=1,2)$ are strongly analytic and $Y_{1}+Y_{2}$ is closed, then $Y_{1} \cap Y_{2}$ is also strongly analytic. The converse of this is false. If $Y_{1}, Y_{2}$, and $Y_{1} \cap Y_{2}$ are strongly analytic, $Y_{1}+Y_{2}$ need not be closed. Radjabalipour [10, Example 3] defines a strongly decomposable operator $T$ for which $X_{T}\left(F_{1}\right)+X_{T}\left(F_{2}\right)$ is not closed, but in this case $X_{T}\left(F_{i}\right),(i=1,2)$ and $X_{T}\left(F_{1} \cap F_{2}\right)=X_{T}\left(F_{1}\right) \cap X_{T}\left(F_{2}\right)$ are strongly analytic. 


\section{REFERENCES}

1. E. Albrecht, On two questions of 1. Colojoara and C. Foias, Manuscripta Math., 25 (1978), 1-15.

2. E. Bishop, A duality theory for an arbitrary operator, Pacific J. Math., 9 (1959), 379-397.

3. I. Erdelyi and S. Wang, A local spectral theory for closed operators, London Math. Soc., Lecture Note Series No 105 (Cambridge University Press, 1985).

4. C. Foias, On the maximal spectral spaces of a decomposable operator, Rev. Roumaine Math. Pures Appl., 15 (1970), 1599-1606.

5. T. Kato, Perturbation theory for linear operators (Springer-Verlag, 1980).

6. R. Lange, Strongly analytic subspaces, in Operator Theory and Functional Analysis, Research Notes in Math. 38, Pitman Advanced Publishing Program, (San Francisco, 1979), 16-30.

7. R. Lange, On generalization of decomposability, Glasgow Math. J., 22 (1981), 77-81. 93-108.

8. R. Lange, Duality and asymptotic spectral decompositions, Pacific J. Math., 121 (1986),

9. R. Lange and S. Wang, New criteria of decomposable operators, Illinois J. Math., to appear.

10. M. Radjabalipour, On decomposition of operators, Michigan Math. J., 21 (1974), $265-275$.

11. M. Radjabalipour, Equivalence of decomposable and 2-decomposable operators, Pacific $J$. Math., 77 (1978), 243-247. $144-158$.

12. G. Shulberg, Decomposable restrictions and extensions, J. Math. Anal. Appl., 83 (1981),

13. J. C. Snader, Bishop's condition $(\beta)$, J. Math. Anal. Appl., in print.

14. S. Wang, A characterization of strongly decomposable operators and its duality theorem, Acta Math. Sinica, 29 (1986), 145-155.

Department of Mathematics

Central Michigan University

Mount Pleasant

MiCHIGAN 48859

U.S.A. 